\title{
MIDAS
}

Museus e estudos interdisciplinares

\section{O impacto das políticas culturais no desenvolvimento de programas para jovens na Tate (1989-2019)}

The impact of cultural policies in the development of youth programs in Tate (1989-2019)

\section{Carolina Silva}

\section{OpenEdition}

\section{Journals}

\section{Edição electrónica}

URL: https://journals.openedition.org/midas/2785

DOI: $10.4000 /$ midas. 2785

ISSN: 2182-9543

\section{Editora:}

Alice Semedo, Paulo Simões Rodrigues, Pedro Casaleiro, Raquel Henriques da Silva, Ana Carvalho

\section{Refêrencia eletrónica}

Carolina Silva, «O impacto das políticas culturais no desenvolvimento de programas para jovens na Tate (1989-2019)», MIDAS [Online], 13 | 2021, posto online no dia 15 dezembro 2021, consultado no dia 05 março 2022. URL: http://journals.openedition.org/midas/2785 ; DOI: https://doi.org/10.4000/ midas. 2785

Este documento foi criado de forma automática no dia 5 março 2022.

\section{(c) (i) (9)}

Midas is licensed under a Creative Commons Attribution-NonCommercial-ShareAlike 3.0 International License 


\section{O impacto das políticas culturais no desenvolvimento de programas para jovens na Tate (1989-2019)}

The impact of cultural policies in the development of youth programs in Tate (1989-2019)

\section{Carolina Silva}

\section{NOTA DO EDITOR}

Artigo recebido a 30.01.2021

Aprovado para publicação a 26.07.2021

Policy statements and documents concerned with the arts or culture may speak volumes, but too often do no more than speak on shelves. (Willis

1990, 52)

\section{Museus e jovens: o caso da Tate}

Os jovens foram desde sempre os outsiders nos públicos dos museus, especialmente se considerados como visitantes independentes - fora do contexto escolar ou de visita em família (Allard 1993; Schwartz 2005). Na perspectiva dos museus, algumas das razões nomeadas para justificar o seu cepticismo em relação aos jovens, são as ideias préconcebidas sobre os desafios de trabalhar com esta faixa etária, a falta de formação das equipas dos museus ou a necessidade de recursos adicionais em termos de espaço, tempo e dinheiro para desenvolver novos programas (LeBlanc 1993; Lemerise 1995; Reeve 2006). A resposta ao porquê e como é que os museus, em particular os museus de arte contemporânea, se voltaram nas últimas décadas para este público, e os deslocaram das margens para o centro da sua programação, não é linear. No entanto, 
uma maior consciência e preocupação com a visibilidade da responsabilidade social do museu, aliada a uma nova visão, mais centrada no visitante, contribuiu para o crescente investimento nos públicos mais jovens (Jackson 2000). Com o intuito de se tornarem mais inclusivos e de reconciliarem compromissos históricos como colecionar, preservar e expor, com um novo entendimento da sua função educativa, os museus começaram a problematizar as lacunas na sua programação, nomeadamente a dirigida aos jovens (Shelnut 1994; Lemerise 1999). ${ }^{1}$

2 A par das mudanças epistemológicas que redefiniram o papel dos públicos nos museus, um impulso sociopolítico levou-os a justificarem o seu financiamento por entidades públicas e/ou privadas (Sandell 1998). No entanto, a definição e implementação das políticas culturais no Reino Unido, em particular as dirigidas aos museus, não é linear e, «até recentemente, em vez de terem sido orientadas por objectivos do governo, têm-se desenvolvido em grande medida em resposta a eventos ou questões» (Selwood 2001, 22, tradução da autora). No caso concreto do investimento na relação dos museus com os jovens, os motins em Meadow Well Estate, Tyneside, em 1990, e mais recentemente os motins de 2011, em Londres, foram factores determinantes na criação de linhas de financiamento para programas com jovens, incluindo em museus (Harland, Kinder e Hartley 1995; Miller 2018). O relatório Moving Culture: An Enquiry into the Cultural Activities of Young People (Willis 1990), comissariado pela Fundação Calouste Gulbenkian (delegação inglesa), foi também um importante alicerce por defender que as instituições culturais, nomeadamente os museus, deveriam desenvolver programas para jovens fora do âmbito da educação formal. ${ }^{2}$ Ao identificar um alto nível de exclusão desta faixa etária das instituições culturais, o impacto deste relatório foi duplo. Por um lado, influenciou a criação de linhas de financiamento dedicadas a projectos de artes para jovens e, por outro lado, gerou novas comissões de investigação e avaliação sobre os recursos para jovens em museus de arte no Reino Unido.

Os programas que os museus oferecem fora do âmbito escolar para jovens foram criados em resposta a premissas específicas, nomeadamente, envolver públicos menos representados, construir as suas futuras gerações de visitantes e/ou tornar os seus espaços mais apelativos para os jovens (Arias e Gray 2007). Das diferentes tipologias de programação disponíveis para esta faixa etária (15-25 anos) destacam-se as iniciativas continuadas, sustentadas numa abordagem de co-criação com e para os jovens. No Reino Unido, o programa Young Tate, criado em 1994 na Tate Liverpool, foi pioneiro na introdução de uma programação que envolve activa e sistematicamente os jovens no museu. Hoje em dia o programa para jovens da Tate assume um perfil de destaque, não só dentro da instituição como a nível nacional e internacional (Miller, Moilliet e Daly 2019).

4 Neste artigo pretende-se analisar o impacto das políticas culturais no desenvolvimento do programa Young Tate entre 1989 e 2019. Esta baliza cronológica, 1989-2019, contempla o lançamento do primeiro programa de jovens na Tate Liverpool - Mobile Art Programme (1989) - e a publicação do relatório final (Miller, Moilliet e Daly 2019) do último grande projecto para jovens da Tate - Circuit (2013-2015). Este intervalo de tempo permite uma análise longitudinal que traça a origem, os desenvolvimentos e os ecos, presentes e futuros, de uma linha programática na Tate. Para o efeito foi realizado o mapeamento dos principais fluxos de influência entre a esfera política, cultural e social, e as práticas do museu, com base na análise de relatórios de avaliação, policy briefs e outras publicações relevantes. 


\section{Políticas culturais e linhas de financiamento no Reino Unido: entre o público e o privado}

5 Observando o panorama político no Reino Unido durante o período abrangido pela presente análise, 1989 a 2019, identificam-se três ciclos distintos. Se entre 1979 e 1996 o partido Conservador liderou o Governo, entre 1997 e 2010 este ciclo de poder foi quebrado pela maioria Trabalhista. Desde então tem sido a frente conservadora a dirigir o parlamento britânico. Esta alternância, pese embora a extensão de cada período governativo, não é em si suficiente para compreender as políticas culturais que afectaram o desenvolvimento de programas de extensão educativa, em particular, os dirigidos aos jovens na Tate ou em outros museus no Reino Unido. Isto deve-se em parte às distintas temporalidades que marcam este domínio. Existe um hiato intrínseco entre o gizar de um documento, a sua operacionalização, nomeadamente através de linhas de financiamento e a acção dos museus. As directrizes políticas tingem os limites das transições governativas e devem ser entendidas como fenómenos multidimensionais (Lang, Reeve e Woollard 2006). Faz por isso sentido considerar os valores epistemológicos, sociais e/ou económicos que informaram o pensamento e a prática das políticas culturais dirigidas aos museus durante este período.

6 A ênfase no compromisso com o acesso, no sentido de abertura dos museus ao público em geral e, em particular, aos grupos socioeconómicos menos representados entre os seus visitantes, é um pilar das políticas culturais para os museus, a par da preocupação em garantir a melhor relação custo-benefício no uso de dinheiros públicos (Creigh-Tyte e Selwood 1998). Para compreender a preocupação com o acesso é necessário abrir este mapeamento às discussões contemporâneas sobre o lugar da educação nos museus. 0 relatório A Common Wealth: Museums and Learning in the United Kingdom (Anderson 1997), comissariado pelo Department for National Heritage (DNH), marca um momento de viragem nas políticas culturais dirigidas aos museus, pelo destaque dado às suas responsabilidades sociais. Para Eilean Hooper-Greenhill, o apelo político à expansão da oferta educativa nos museus «foi motivado pela conviç̧ão ideológica do governo [trabalhista] de que a cultura devia ser socialmente inclusiva, responsável e usada mais pelas escolas» (Hooper-Greenhill 2007, 6, tradução da autora). A aceitação explícita da função educativa dos museus possibilitou também o acesso a novas linhas de financiamento. ${ }^{3}$

7 No que respeita à relação custo-benefício no uso de dinheiros públicos, destacam-se dois indicadores: a crescente produção de estudos sobre públicos - actuais e prospectivos, e a introdução de parâmetros mensuráveis de sucesso na avaliação do desempenho dos museus (Creigh-Tyte e Selwood 1998). O primeiro indicador pretende alicerçar a decisão de investimento em determinados segmentos de público. Olhando para a relação entre museus e jovens, destacam-se quatro publicações determinantes no desenvolvimento de programas fora do âmbito escolar para jovens em museus, nomeadamente na Tate: Moving Culture: An Enquiry into the Cultural Activities of Young People (Willis 1990), Arts in Their View: A Study of Youth Participation in the Arts (Harland, Kinder e Hartley 1995), Enquiry into Young People and Art Galleries (Selwood, Clive e Irving 1995) e Crossing the Line: Extending Young People's Access to Cultural Venues (Harland e Kinder 1999). Apesar de pautados por objetivos e metodologias distintas, todos evidenciam o distanciamento existente entre museus e jovens, e assinalam os benefícios 
bilaterais desta relação, nomeadamente, a importância do reconhecimento das práticas culturais próprias dos jovens no diálogo com sistemas artísticos mais convencionais, como os representados nos museus.

Relativamente ao segundo indicador, a introdução de parâmetros mensuráveis de sucesso na avaliação do desempenho dos museus, com destaque para a contagem de visitantes e participantes em actividades, favorece uma análise quantitativa em detrimento de uma leitura qualitativa destas experiências. No caso da Tate, a introdução de indicadores de performance no relatório anual, incluindo a segmentação de participantes por faixas etárias imposta pelo Department for Digital, Culture, Media and Sport (DCMS) $)^{4}$, teve impacto na programação educativa no seu todo, e na dirigida aos jovens, em particular, pelo favorecimento de iniciativas de grande escala em detrimento de programas continuados (Sayers 2014b). A dicotomia quantidade versus qualidade é sintomática de entendimentos contraditórios do papel do museu. Valores como popularidade, diversidade e excelência existem em tensão nas políticas e práticas culturais no Reino Unido (Street 2011). No entanto, a sua conciliação é vital para a sustentabilidade financeira e programática dos museus.

Importa enquadrar sumariamente o contexto de financiamento do sector cultural britânico, em particular o que afecta os museus, para compreender e problematizar de forma efectiva as suas políticas culturais. Os maiores pacotes de financiamento para os museus são assegurados pelo governo central através do DCMS, das autoridades locais e da National Lottery (Selwood 2001). ${ }^{5}$ Por exemplo, o DCMS financia directamente 17 museus nacionais através de subsídios, incluindo a Tate. Uma das prioridades deste apoio é garantir que o acesso aos museus é gratuito. ${ }^{6}$ No entanto, esse apoio não representa a totalidade do orçamento de cada museu, sendo que no caso da Tate cerca de $70 \%$ do seu financiamento anual é assegurado por fontes não-governamentais. ${ }^{7} \mathrm{Sem}$ pretensão de apresentar uma descrição exaustiva das políticas e linhas de financiamento do sector cultural no Reino Unido, irei mapear os seus principais intervenientes, públicos e privados, e a forma como influenciaram e influenciam o funcionamento dos museus, em particular, na sua extensão educativa e trabalho com jovens. Destaco assim o contributo do Arts Council, da Fundação Calouste Gulbenkian (delegação inglesa) e da Fundação Paul Hamlyn.

\section{Triângulo de ouro: Arts Council, Fundação Calouste Gulbenkian e Fundação Paul Hamlyn}

10 Em 1964 Jennie Lee foi nomeada Ministra das Artes pelo Governo trabalhista, sendo autora do primeiro white paper sobre as artes, A Policy for the Arts: First Steps (1965). O documento reconheceu a importância de todas as pessoas terem acesso igual às artes, em todo o país, e criou um precedente importante para as intervenções dos governos seguintes na área das artes e na sua articulação com a educação (Doeser 2015). ${ }^{8} \mathrm{~A}$ responsabilidade da execução das directrizes esboçadas pelo Governo foi colocada no Arts Council, assente no princípio de que «se desde cedo as crianças se habituarem à ideia da arte como parte da vida quotidiana, é mais provável que na maturidade, primeiro, as aceitem e, depois, as exijam» (Lee 1965, 14, tradução da autora). Como o título do plano estratégico do Arts Council indica, Achieving Great Art for Everyone: A Strategic Framework for the Arts (Arts Council England 2010), 40 anos depois, o princípio 
de financiar o melhor e torná-lo acessível à maioria manteve-se como orientador das políticas e práticas de financiamento culturais no Reino Unido. ${ }^{9}$

11 Criado em 1946, o Arts Council deriva do Council for Encouragement of Music and Art, estabelecido no Reino Unido durante a Segunda Guerra Mundial com o propósito de levar as artes a lugares isolados do resto da sociedade..$^{10}$ Apesar das diversas alterações que sofreu ao longo dos anos, o Arts Council é ainda hoje um dos principais decisores das políticas e do financiamento do sector cultural. A sua génese teve como objetivo a criação de um organismo que distribuísse dinheiro público às artes com base na recomendação de peritos e sem qualquer interferência política (Redcliffe-Maud 1976). Embora seja um organismo público não departamental do DCMS, o Arts Council tem autonomia para alocar recursos a projectos, artistas e/ou instituições culturais. Entre 2018 e 2022, o National Portfolio do Arts Council England (ACE) inclui 827 instituições culturais, das quais 57 são museus, que irão receber um total de 71.3 milhões de libras de apoio ao seu funcionamento, nomeadamente para o desenvolvimento de programas educativos, uma área que nem sempre foi considerada prioritária. ${ }^{11}$

O relatório Support for the Arts in England and South Wales (1976), publicado pela Fundação Calouste Gulbenkian (delegação inglesa) e comissariado pela Standing Conference of Regional Arts Associations ${ }^{12}$ e pelo Arts Council, teve como objectivo analisar as estruturas de financiamento das artes no Reino Unido. Entre as conclusões e as recomendações destaca-se a referência ao papel da educação no mecenato artístico:

[...] temos de rejeitar a falácia enraizada de que o 'apoio às artes' e a 'educação' são duas coisas separadas. Devemos insistir, mais positivamente, que aqueles que são responsáveis por ambos são aliados naturais, e garantir que eles colaboram a nível nacional, regional e local. (Redcliffe-Maud 1976, 23, tradução da autora)

13 Uma figura central nesta mudança de paradigma no seio do Arts Council foi Roy Shaw, Secretário-Geral entre 1975 e 1983, e responsável pela nomeação dentro do Arts Council da primeira Education Liaison Officer, Irene MacDonald, em 1978, e em 1979 da primeira Art Education Officer, Pat van Pelt. Ambas as posições foram financiadas pela Fundação Calouste Gulbenkian (delegação inglesa), uma aliada de peso no debate sobre artes e educação no Reino Unido.

Criada em 1956, a Fundação Calouste Gulbenkian (delegação inglesa), publica o seu primeiro relatório em 1959, Help for the Arts (Calouste Gulbenkian Foundation 1959) com o intuito de mapear as necessidades das artes no Reino Unido no clima de pós-guerra e identificar o papel que poderia ter. Desde então actua activamente através de linhas de financiamento na área das artes e da cultura, do bem-estar social e da educação, assim como da publicação de relatórios e recomendações, com um legado significativo nas políticas e práticas no contexto britânico (Doeser 2015).

15 Operando igualmente como uma organização independente, a Fundação Paul Hamlyn foi criada em 1987 e herda o nome do seu fundador. Defende na sua visão «uma sociedade justa na qual todos, especialmente os jovens, possam alcançar o seu potencial máximo e ter vidas plenas e criativas» (Paul Hamlyn Foundation 2021, para. 1). Para a sua concretização disponibiliza diferentes linhas de apoio e financiamento, nomeadamente para organizações que trabalham com jovens, tanto no sector social, como na educação e/ou na cultura. Destaca-se, pela relevância no trabalho dos museus com jovens, o Arts and Participation Fund, que oferece bolsas a projectos que abordam e procuram resolver de forma estrutural as desigualdades nas oportunidades de acesso às 
artes. Ambas as fundações tiveram um papel crucial no lançamento, desenvolvimento e expansão do programa para jovens na Tate.

\section{Tate: as idiossincrasias programáticas de uma instituição multiespacial}

16 A Tate inaugurou em 1897, e, desde então cresceu, sendo agora uma instituição multiespacial que reúne a colecção nacional de arte britânica e de arte moderna internacional e contemporânea. Deve o seu nome a Henry Tate (1819-1899), um homem da indústria que doou a sua colecção privada de arte ao país, guardada num edifício desenhado propositadamente em Millbank, Londres (Spalding 1998). Conhecida actualmente como Tate Britain, integra uma família de quatro galerias de arte - Tate Liverpool, Tate St Ives e Tate Modern, inauguradas, respectivamente, em 1988, 1993 e $2000 .{ }^{13} \mathrm{O}$ papel da educação na Tate cresceu a par da sua expansão física, embora só em 1969 tenha sido criada a primeira equipa educativa, e, um ano depois, fundado o Departamento de Educação e Exposições. ${ }^{14}$

exposições, uma decisão que abriu discretamente caminho à sua visibilidade dentro da instituição, e que influenciou tacitamente a criação de um Departamento Educativo na Tate Liverpool desde o início. 30 anos depois foi lançado o programa Transforming Tate Learning, uma iniciativa que pretendia colocar a educação (learning) no centro da instituição (Cutler 2010; Pringle 2013). Esta viragem encetou não só uma mudança de estratégia organizacional, mas, e talvez acima de tudo, uma mudança epistemológica que ecoa e expande os debates teóricos na área da educação em museus. ${ }^{15}$

18 Ancorado num projecto de investigação-acção financiado pela Fundação Paul Hamlyn, que decorreu durante três anos (2010-2013), o programa Transforming Tate Learning defende:

[...] uma abordagem mais participativa e centrada no visitante (learner), mais preocupada com a criação de contextos de aprendizagem e de oportunidades para os públicos interagirem activamente com a arte, e não tanto com a transmissão de conhecimentos especializados sobre obras de arte. (Pringle e DeWitt 2014, s/p, tradução da autora)

Um dos pilares desta mudança foi a ênfase numa prática informada pela investigação (research-led practice), em que educadores do museu, artistas e participantes partilham processos criativos e de reflexão crítica. Anna Cutler, Directora de Learning and Research da Tate, reconhece o impacto deste processo, tanto nas metodologias e nas tipologias de programação, como na visibilidade interna dada ao trabalho da equipa educativa (Cutler n.d.).

Em 2016 inicia-se uma nova torção na história da Tate com a criação do programa educativo Tate Exchange, desenvolvido na Tate Modern, na Tate Liverpool e em formato online. Definido como um «programa participativo aberto, de atividade e experimentação» (Cutler 2018, tradução da autora), é enquadrado numa temática anual e pretende juntar o museu e as suas instituições parceiras, com artistas e visitantes através de encontros criativos de debate. A programação do Tate Exchange está estruturada em três momentos: o primeiro inclui a programação pela equipa da Tate de actividades como debates, workshops ou eventos; o segundo é programado pelas instituições parceiras; e o terceiro é dedicado à avaliação dos anteriores (Christensen- 
Scheel 2018). Foi por entre estas sucessivas viragens no percurso institucional e na programação educativa da Tate que se desenhou o seu programa para jovens.

\section{O programa Young Tate}

20 Apesar da sua consanguinidade com a Tate Britain, a Tate Liverpool moldou um caminho único de extensão, incluindo um programa pioneiro para jovens. As características pós-industriais e sociodemográficas da cidade de Liverpool influenciaram a identidade da galeria desde o início e determinaram a sua missão principal - encorajar a captação de novos públicos, através de um programa educativo activo (Ganga 2009). Sob a supervisão de Toby Jackson, Director Educativo entre 1988 e 1999, altura em que foi para a Tate Modern, em Londres, a equipa educativa combinou esforços para planear e desenvolver atividades em diálogo com as exposições, assim como trabalho fora da galeria, de forma a alcançar os seus não-visitantes (Jackson 2000). Uma das estratégias adoptadas foi uma abordagem de marketing «dirigida a grupos específicos com um programa contínuo de eventos, em que cada programa tinha um curador educativo (Education Curator), uma estratégia de marketing e métodos e recursos pedagógicos específicos» (Jackson 2000, 25, tradução da autora). Foi neste contexto que em 1989 surgiu o Mobile Art Programme (MAP), uma iniciativa de extensão, fora da educação formal, dirigida a jovens entre os 14 e os 25 anos.

21 A publicação Testing the Water: Young People and Galleries, editada por Naomi Horlock (2000), coordenadora do programa Young Tate entre 1988 e 2007, oferece uma perspectiva aprofundada da vida, ou vidas, do programa. Através de workshops pontuais na galeria, que envolviam os participantes em discussões críticas sobre as obras em exposição, o principal objetivo do MAP foi desenvolver ligações entre a cultura juvenil e a cultura do museu. $O$ contexto para testar e desenvolver novas metodologias para esta faixa etária foi possível devido ao financiamento de dois anos da Fundação Calouste Gulbenkian (delegação inglesa) ${ }^{16}$ e informado pela colaboração com organizações juvenis e pelo testemunho continuado dos participantes.

22 Ao longo dos anos tornou-se claro que o impacto de actividades pontuais era limitado. Com base nessa experiência e nas ideias partilhadas no Youth Arts Weekend, um evento organizado no verão de 1993 para envolver jovens na discussão da sua futura participação na galeria, foi criado, em 1994, o programa Young Tate. A passagem para um compromisso a longo prazo «coincide com a revisão, pelo Departamento Educativo, do papel do trabalho de extensão em relação à programação in-house» (Horlock 2000, 50, tradução da autora). Consequentemente, o programa para jovens passou a estar mais interligado com as práticas curatoriais e com a programação de exposições.

o Young Tate começou por ser um grupo consultivo, cujo principal objectivo era informar o museu sobre como tornar a sua colecção mais atraente para os visitantes mais jovens. Passado um ano do projecto-piloto, e em resposta à avaliação dos participantes, houve uma mudança na dimensão consultiva inicial em direção a um envolvimento mais inclusivo dos jovens no planeamento e no desenvolvimento de atividades para os seus pares, nomeadamente workshops com artistas, eventos e visitas guiadas. A abordagem entre pares - com e para jovens - foi testada gradualmente, e, ao fim do terceiro ano, o ethos do programa estava «firmemente centrado no desenvolvimento contínuo de trabalho peer-led, e na profissionalização da abordagem da Galeria à formação e na melhoria da qualidade das experiências dos jovens» 
(Horlock 2000, 125, tradução da autora). Depois de a galeria abrir ao público foi introduzido um curso de formação para jovens, desenhado em colaboração com organizações juvenis parceiras, antigos membros do programa e pessoal da galeria. Neste âmbito foi desenhado um perfil específico para os membros do Young Tate, o de peer-leader, que ainda hoje informa os programas para jovens da Tate.

\section{O programa Raw Canvas}

O programa Raw Canvas foi criado em 1999 na Tate Modern. Apesar de ter sido influenciado pela estrutura, metodologia e valores do Young Tate, este programa foi desenvolvido para responder ao público local e, por essa razão, estabeleceu a sua própria identidade (Sayers 2011). Um dos objetivos foi «formar jovens consumidores culturais de diversos contextos [socioculturais] e assim promover um interesse pela arte ao longo da vida de forma a mudar a demografia dos visitantes adultos dos museus no futuro» (Sayers 2011, 140, tradução da autora). Uma característica única do Raw Canvas é que começou antes da galeria abrir ao público e, assim, respondeu simultaneamente a uma estratégia de extensão, de formação de públicos e de marketing.

A experiência do projecto-piloto fundamentou uma candidatura à Fundação Paul Hamlyn, que apoiou o programa até $2005 .{ }^{17}$ Enquadrado por uma agenda política e institucional inclusiva, o programa foi desenhado com e para jovens, com idades entre os 15 e os 23, que podiam participar em eventos de larga escala, workshops ou outras atividades desenvolvidas pelos peer-leaders do Raw Canvas (Sayers 2014a). ${ }^{18} \mathrm{Na}$ outra margem do rio Tamisa, a alguns quilómetros de distância, a Tate Britain criou o seu próprio colectivo de jovens em 2002, chamado Tate Forum, e em 2006 foi estabelecido o programa Young Tate St Ives na Cornualha.

Embora acomodando a especificidade de cada programa, informada em parte pela demografia de cada galeria, em 2006 os curadores dos programas para jovens (youth curators) da Tate criaram um conjunto de objectivos, com base na sua experiência local e planos futuros, que deveriam ser partilhados entre as quatro galerias.

objectivo 1: garantir benefícios a longo prazo para jovens que já estão interessados em cultura visual, atrair os que não estão, e melhorar as vidas e potencial de saídas profissionais de todos os participantes no Young Tate, através do seu envolvimento mais aprofundado e variado na Tate e nas suas galerias locais.

Objectivo 2: criar um espaço para a troca de novas ideias no qual os jovens são consultados, têm oportunidades de participar no processo cultural da Tate e podem ter controlo sobre as suas aprendizagens.

Objectivo 3: ser inclusivo e diversificado no conteúdo do programa e nos jovens que participam nestes programas. (Sinker 2008, tradução da autora)

Esta confluência foi consolidada ainda mais quando, em 2010, o Raw Canvas e o Tate Forum se fundiram num único colectivo de jovens - o Tate Collective, um nome adoptado mais tarde pelas outras duas galerias da Tate.

\section{O programa Circuit}

Sustentado em mais de 20 anos de experiência de trabalho com jovens, o programa Circuit, lançado em 2013 e concluído em 2017, ilustra o crescimento da ambição da Tate, pela escala e geografia da sua extensão. Financiado pela Fundação Paul Hamlyn, com um apoio de cinco milhões de libras, envolveu, além das quatro galerias da Tate, outros 
seis museus e galerias no Reino Unido: Firstsite (Colchester), MOSTYN (Llandudno), Nottingham Contemporary, Whitworth Art Gallery (Manchester), Wysing Arts Centre e Kettles Yard (ambos em Cambridgeshire) ${ }^{19}$, juntamente com mais de 80 parceiros de diferentes sectores, incluindo da juventude e educação, instituições de caridade e autoridades locais. Dirigido a jovens entre os 15 e os 25 anos, o programa teve como principais objectivos:

Fazer uma diferença positiva com e para os jovens.

Melhorar o acesso e oportunidades para jovens mais difíceis de envolver, através da extensão e desenvolvimento de redes sustentáveis entre os sectores das artes e da juventude.

Desenvolver e mudar as práticas dentro e entre instituições culturais.

Mudar as atitudes e os comportamentos em relação a e sobre os jovens. (Miller, Moilliet e Daly 2019, 13, tradução da autora)

A complexidade e os desafios inerentes à concretização deste projecto estão expressas nas publicações Circuit: Test, Risk, Change: Young People, Youth Organisations and Galleries Working Together (Miller, Moilliet e Daly 2019) e Youth Work, Galleries and the Politics of Partnerships (Sim 2019). ${ }^{20}$

Das quatro linhas que orientaram o programa - parcerias, peer-led, festival e digital destaca-se, pela sua relevância para o tópico em análise, as duas primeiras. A colaboração entre o sector cultural e os sectores terciários, nomeadamente a educação e a atividade social, é um objetivo charneira da Fundação Paul Hamlyn (Cochefert 2019). 0 enfoque no trabalho em rede, tanto entre museus e galerias como entre estes e outras instituições, serve também o interesse da Tate na problematização do seu papel enquanto instituição cultural pública. Os modelos testados através do Circuit assinalam a importância de encontrar objetivos e linguagens comuns, por forma a tornar sustentáveis as parcerias interinstitucionais e não comprometer os princípios éticos implícitos na relação com os jovens, alguns dos quais em situação de risco (Sim 2019). A opção por uma metodologia peer-led dá continuidade ao trabalho feito pela Tate, que replica as abordagens usadas em outros programas para jovens em museus de arte contemporânea. ${ }^{21} \mathrm{O}$ perfil de peer-leader parece, no entanto, cruzar-se com o de produtor cultural. Neste sentido, os jovens são vistos não só como co-organizadores de eventos para os seus pares, mas também como produtores culturais - artistas visuais, músicos, poetas, curadores, com uma voz criativa própria. Exemplo dessa mudança é a alteração recente do nome do grupo Tate Collective para Tate Collective Producers. ${ }^{22}$

\section{Em discussão: pontos de tensão e fluxos de influência}

29 A análise do programa para jovens da Tate, à luz do desenvolvimento das políticas culturais no Reino Unido, entre 1989 e 2019, revela um panorama complexo, no qual as relações de influência não são biunívocas. É possível identificar momentos em que políticas e linhas de financiamento concretas alavancaram a programação fora do âmbito escolar para jovens na Tate como, por exemplo, o apoio da Fundação Calouste Gulbenkian (delegação inglesa) ao Mobile Art Programme na Tate Liverpool, ou o financiamento da Fundação Paul Hamlyn nos primeiros anos do Raw Canvas, na Tate Modern. No entanto, a afirmação da programação para jovens dentro da Tate é, tanto ou mais, fruto do trabalho continuado de vários interlocutores - jovens, educadores, artistas, diretores e instituições parceiras. A capacidade de adaptação de todos permitiu testar, avaliar e ajustar estratégias, assim como tipologias de programação em resposta 
a tensões externas e internas. Este não foi um percurso linear, mas pode ser considerado um percurso de sucesso, expresso na gradual deslocação de um programa de extensão, realizado fora de portas, para um programa continuado central a todo o projecto educativo e ao perfil institucional da Tate.

Se o que enquadrou as primeiras iniciativas para jovens em museus foi um discurso de responsabilização social perante grupos menos representados nos seus públicos, nomeadamente jovens em situação de risco de exclusão social, o caminho traçado ao longo dos últimos 30 anos foi de autonomização. A programação para jovens na Tate, assim como em outros museus de arte contemporânea, é hoje válida e relevante por si. Embora mantendo um compromisso com práticas inclusivas, que procuram chegar a todos os jovens, é reconhecida não só a individualidade criativa de cada um, como a sua capacidade de trabalho em equipa - com os seus pares e o museu, na produção de conteúdos e de programas dinâmicos. O sucesso desta emancipação, no caso da Tate, é expresso na escala e na ambição do programa Circuit, cujo impulso combina tanto a experiência e a vontade do museu como os interesses da entidade financiadora, a Fundação Paul Hamlyn. Desenha-se, assim, um fluxo de influência, que abre caminho a um processo de experimentação partilhado, onde há lugar para o erro, ou seja, a prática não é pré-determinada por premissas e políticas fechadas, mas por questões abertas.

Do contributo do percurso da Tate na programação para jovens, em linha com o seu projecto educativo, destaca-se o reconhecimento do papel da investigação participativa (Pringle 2019). No caso do trabalho com e para jovens, a centralidade das suas vozes na definição, criação e avaliação das actividades que lhes são dirigidas foi crucial para a sua sustentabilidade. A visibilidade destes processos foi igualmente relevante, em particular pela sua amplitude, o que serve tanto um princípio de acessibilidade como as estratégias de comunicação e de desenvolvimento do próprio museu. Incluiu, ao longo de 30 anos, livros, relatórios, artigos, teses de doutoramento, conferências, grupos públicos de discussão, blogues, entre outros. A exigência por parte das entidades públicas e privadas que financiam o sector cultural no Reino Unido de estudos prospectivos e/ou de relatórios de avaliação, acompanhada pelo apoio de posições integradas nos museus para investigadores e/ou estudantes de doutoramento, é também um princípio fundador de boas práticas presentes e, acima de tudo, futuras. 0 conhecimento produzido pelo e com os museus e os seus interlocutores - públicos, instituições parceiras, artistas - deve ser um alicerce de referência no desenho das políticas culturais.

O testemunho do longo e estabelecido percurso do programa de jovens da Tate mostra como a sua sustentabilidade está intrinsecamente ligada a um compromisso de fundo da instituição com o seu projecto educativo e social. O sucesso de projectos pontuais e desassociados da missão do museu está mais condicionado, particularmente quando o público-alvo são jovens:

Um dos pontos chave a considerar sobre a vossa organização é porque é que querem

atrair jovens... é simplesmente porque esse é um objectivo do governo? Mas lembrem-se: os jovens conseguem ouvir o som do tokenismo... Se a instituição não estiver no seu todo por trás dessa decisão, os jovens irão afastar-se... (Ings 2001, 34, citado em Reeve 2006, 52, tradução da autora)

Dar voz aos participantes na concepção, desenvolvimento e avaliação dos programas que lhes são dirigidos é crucial para um envolvimento crítico. ${ }^{23}$ Ao focar o impacto das políticas culturais no desenvolvimento de programas para jovens da Tate, o presente mapeamento privilegiou o discurso institucional em detrimento das vozes dos 
participantes, embora se reconheça o seu valor e potencial. Pensar em formas de trazer estes testemunhos para o território da avaliação e dos estudos de público, sem os instrumentalizar, é uma problemática de investigação futura. Olhar para os números a par dos percursos que contam individualmente.

\section{Nota conclusiva}

O futuro do sector cultural apresenta-se hoje sob um fundo de enorme incerteza. Os estudos revelam o impacto significativo da actual pandemia covid-19 nos museus (NEMO 2021). Sublinha-se, porém, a resiliência e a capacidade de resposta dos programas para jovens em museus de arte contemporânea (Silva 2021). A transição da sua atividade para o plano virtual deu origem a vários eventos, em que se destacam: Late at Tate Britain Online (15 Dezembro, 2020), organizado pelo grupo Tate Collective Producers; Pause: A Digital Teen Night (17 Abril 2020), organizado pelo grupo de jovens do Museu de Arte Contemporânea de Los Angeles; GENEXT Goes Online (31 Maio, 2020), produzido pelo comité de jovens do Museu de Arte Contemporânea da Austrália; ou à exposição Home: Live In Room (Agosto 2020), com a curadoria do colectivo de jovens da Whitechapel Gallery. Estes são apenas alguns exemplos aos quais muitos outros se poderiam juntar. Revelam todos o resultado de um investimento continuado por parte dos museus, embora nem sempre em consonância com directrizes políticas, em programas com e para jovens. Só esse lastro torna possível pensar os tempos por vir com alguma confiança.

\section{BIBLIOGRAFIA}

Allard, Michel. 1993. “Les Adolescents et les Musées.” Revue des Sciences de l'Éducation 19 (4): 766-774.

Allen, Felicity. 2008. "Situating Gallery Education.” Tate Encounters 2 (1): 1-12.

Anderson, David. 1997. A Common Wealth: Museums and Learning in the United Kingdom: A Report to the Department of National Heritage. London: Department of National Heritage.

Arias, Catherine, e Denise A. Gray. 2007. "Adolescents in Art Museum: Key Considerations for Successful Programs." In From Periphery to Center: Art Museum Education in the $21^{\text {st }}$ Century, editado por Pat Villeneuve, 96-102. Reston, Virginia: National Art Education Association.

Arts Council England. 2010. Achieving Great Art for Everyone: A Strategic Framework for the Arts. London: Arts Council England.

Burnham, Rika, e Elliott Kai-Kee, eds. 2011. Teaching in the Art Museum: Interpretation as Experience. Los Angeles California: Getty Publications.

Calouste Gulbenkian Foundation, ed. 1959. Help for the Arts: A Report to the Calouste Gulbenkian Foundation. London: Calouste Gulbenkian Foundation (United Kingdom \& British Commonwealth Branch). 
Cawley-Gelling, India, Steven Hyland, Connah McCarron-Roberts, Sufea Mohamad Noor, e Ruth Morrisey. 2015. "The Value of the Blueprint Festival." International Journal of Art and Design Education 34 (3): 319-325.

Christensen-Scheel, Boel. 2018. "An Art Museum in the Interest of Publicness: A Discussion of Educational Strategies at Tate Exchange." International Journal of Lifelong Education 37 (1): 103-119.

Cochefert, Régis. 2019. “Forward.” In Circuit: Test, Risk, Change: Young People, Youth Organisations and Galleries Working Together, editado por Mark Miller, Rachel Moilliet e Eileen Daly, 8-9. London: Tate e Paul Hamlyn Foundation.

Creigh-Tyte, Stephen W., e Sara Selwood, 1998. "Museums in the UK: Some Evidence on Scale and Activities." Journal of Cultural Economics 22 (2/3): 151-165.

Cutler, Anna. 2010. "What Is to Be Done, Sandra? Learning in Cultural Institutions of the TwentyFirst Century.” Tate Papers 13 (Spring). https://www.tate.org.uk/research/publications/tatepapers/13/what-is-to-be-done-sandra-learning-in-cultural-institutions-of-the-twenty-firstcentury

Cutler, Anna. 2018. “The Value of Values: Reflections on Tate Exchange.” Tate Papers 30 (Autumn). https://www.tate.org.uk/research/publications/tate-papers/30/reflections-on-tate-exchange

Cutler, Anna. n.d. “Tate Learning: Vision and Practice." Consultado em Agosto 16, 2021. https:// www.tate.org.uk/research/research-centres/tate-research-centre-learning/working-papers/ arts-learning-tate

Doeser, James. 2015. Step by Step: Arts Policy and Young People 1944-2014. London: King's College.

Fisher, Rod, ed. 2020. Country Profile: United Kingdom: England and Wales. London: Compendium of Cultural Policies and Trends.

Fróis, João Pedro. 2008. "Os Museus de Arte e a Educação: Discursos e Práticas Contemporâneas.” Museologia.pt 2: 62-75.

Ganga, Rafaela. 2009. "Deindustrialisation, Culture and Education: A Case-Study of Tate Liverpool's Youth-Programme.” The Journal of Learning 16 (9): 481-493.

Harland, John, e Kay Kinder. 1999. Crossing the Line: Extending Young People's Access to Cultural Venues. London: Calouste Gulbenkian Foundation.

Harland, John, Kay Kinder, e Kate Hartley. 1995. Arts in Their View: A Study of Youth Participation in the Arts. Berkshire: National Foundation for Educational Research.

Hooper-Greenhill, Eilean. 2007. Museums and Education: Purpose, Pedagogy, Performance. London: Routledge.

Horlock, Naomi, ed. 2000. Testing the Water: Young People and Galleries. Liverpool: Liverpool University Press e Tate Gallery Liverpool.

Jackson, Toby. 2000. "With Visitors in Mind: The Museum with Permeable Walls." In Testing the Water: Young People and Galleries, ed. Naomi Horlock, 22-30. Liverpool: Liverpool University Press e Tate Gallery Liverpool.

Lang, Caroline, John Reeve, e Vicky Woollard. 2006. "The Impact of Government Policy.” In The Responsive Museum: Working with Audiences in the Twenty-First Century, editado por Caroline Lang, John Reeve e Vicky Woollard, 19-28. Hampshire, England: Ashgate.

LeBlanc, Suzanne. 1993. "Lost Youth: Museums, Teens and the YouthALIVE! Project.” Museum News 72 (6): 44-46. 
Lee, Jennie. 1965. A Policy for the Arts: The First Steps. London: Her Majesty's Stationery Office.

Lemerise, Tamara. 1995. "The Role and Place of Adolescents in Museums: Yesterday and Today." Museum Management and Curatorship 14 (4): 393-408.

Lemerise, Tamara. 1999. “Changes in Museums Benefit Adolescents.” Curator 42 (1): 7-11.

Linzer, Danielle, e Mary Ellen Munley. 2015. Room to Rise: The Lasting Impact of Intensive Teen Programs in Art Museums. New York: Whitney Museum of American Art.

Miller, Mark, Rachel Moilliet e Eileen Daly, eds. 2019. Circuit: Test, Risk, Change: Young People, Youth Organisations and Galleries Working Together. London: Tate, Paul Hamlyn Foundation.

Miller, Mark. 2018. “Introduction.” In Circuit: Test, Risk, Change: Young People, Youth Organisations and Galleries: Working with as Allies to Spark Change, organizado por CIRCUIT, 12-14. London: Tate e Paul Hamlyn Foundation.

Miller, Mark. 2019. “Introduction." In Circuit: Test, Risk, Change: Young People, Youth Organisations and Galleries Working Together, editado por Mark Miller, Rachel Moilliet e Eileen Daly, 10-12. London: Tate e Paul Hamlyn Foundation.

NEMO. 2021. "Follow-up Survey on the Impact of the COVID-19 Pandemic on Museums in Europe: Final Report." NEMO (Network of European Museum Organisations). https://www.ne-mo.org/ fileadmin/Dateien/public/NEMO_documents/NEMO_COVID19_FollowUpReport_11.1.2021.pdf

Paull Hamlyn Foundation. 2021. “Our Vision and Mission”. Consultado em janeiro 20, 2021. https://www.phf.org.uk/reader/strategy-2020/our-vision-and-mission/

Pringle, Emily, e Jennifer DeWitt. 2014. "Perceptions, Processes and Practices Around Learning in an Art Gallery." Tate Papers 22 (Autumn). https://www.tate.org.uk/research/publications/tatepapers/22/perceptions-processes-and-practices-around-learning-in-an-art-gallery

Pringle, Emily, ed. 2013. Transforming Tate Learning. London: Tate.

Pringle, Emily. 2019. Rethinking Research in the Art Museum. London: Routledge.

Redcliffe-Maud, John. 1976. Support for the Arts in England and Wales. London: Calouste Gulbenkian Foundation United Kingdom Branch.

Reeve, John. 2006. "Prioritizing Audience Groups." In The Responsive Museum: Working with Audiences in the Twenty-First Century, editado por Caroline Lang, John Reeve e Vicky Woollard, 43-60. Hampshire, England: Ashgate.

Roberts, Lisa. 1997. From Knowledge to Narrative: Educators and the Changing Museums. Washington, DC: Smithsonian Institution.

Sandell, Richard. 1998. "Museums as Agents of Social Inclusion." Museum Management and Curatorship 17 (4): 401-418.

Sayers, Esther. 2011. "Investigating the Impact of Contrasting Paradigms of Knowledge on the Emancipatory Aims of Gallery Programmes for Young People." International Journal of Art and Design Education 30 (3): 409-422.

Sayers, Esther. 2014a. “'Equality of intelligences': Exploring the Barriers to Engagement in Modern and Contemporary Art Through a Peer-to-Peer Workshop Art Tate Modern." International Journal of Art and Design Education 33 (3): 355-364.

Sayers, Esther. 2014b. “Making 'Culture Vultures': An Investigation into the Socio-Cultural Factors that Determine What and How Young People Learn in the Art Gallery." PhD in Education, Goldsmiths College, University of London. 
Schwartz, Deborah. 2005. "Dude, Where is My Museum? Inviting Teens to Transform Museums." Museum News, 84 (5): 36-41.

Selwood, Sara, Sue Clive, e Diana Irving. 1995. An Enquiry into Young People and Art Galleries. London: Art \& Society.

Selwood, Sarah, ed. 2001. The UK Cultural Sector: Profile and Policy Issues. London: Policy Studies Institute.

Shelnut, Stacey. 1994. "Long-Term Museum Programs for Youth." Journal of Museum Education. 19 (3): 10-13.

Silva, Carolina. 2021. "Pockets of Resilience: The Digital Responses of Youth Collectives in Contemporary Art Museums During Lockdown.” Journal of Museum Education 46 (4): 493-508.

Sim, Nicola. 2018. “'Like Oil and Water'? Partnerships Between Visual Art Institutions and Youth Organisations." PhD thesis, University of Nottingham.

Sim, Nicola. 2019. Youth Work, Galleries and the Politics of Partnerships. Cham: Palgrave Macmillan.

Sinker, Rebecca. 2008. "On the Evolution of a Peer-Led Programme: Tate Forum." Tate Papers 10 (Autumn). Tate Papers. https://www.tate.org.uk/research/publications/tate-papers/10/on-theevolution-of-a-peer-led-programmme-tate-forum

Spalding, Frances. 1998. The Tate: A History. London: Tate Gallery.

Street, John. 2011. "The Popular, the Diverse and the Excellent: Political Values and UK Cultural Policy." International Journal of Cultural Policy 17 (4): 380-393.

Tate Gallery. 2020. “Tate Education History.” Consultado em Novembro 26, 2021. https:// www.timetoast.com/timelines/tate-education-history

Willis, Paul. 1990. Moving Culture: An Enquiry into the Cultural Activities of Young People. London: Calouste Gulbenkian Foundation.

\section{NOTAS}

1. A autora escreve de acordo com a antiga ortografia.

2. 0 estudo que sustenta este relatório teve início em 1987, altura em que o apoio da Fundação Calouste Gulbenkian a actividades criativas para jovens se expandia para além do domínio da escola, contexto em que se tinha centrado até à data (Willis 1990).

3. Um exemplo foi o acesso ao New Opportunities Fund, criado em 1998, para distribuir fundos da Lotaria Nacional a projectos nas áreas da saúde, da educação e do ambiente. https:// www.gov.uk/government/organisations/new-opportunities-fund (consultado em Janeiro 11, 2021).

4. O DCMS foi criado em 1997 e substituiu o Department of National Heritage (DNH). Em 2017 foi renomeado Department for Digital, Culture, Media and Sport. https://www.gov.uk/government/ news/change-of-name-for-dcms (consultado em Setembro 20, 2021).

5. A National Lottery foi criada em 1993 para gerar «dinheiro para boas causas (incluindo as artes) e, especificamente elementos não financiados tradicionalmente através de Grant in Aid (ex. fundos governamentais gerados pelos impostos)» (Doeser 2015, 15, tradução da autora).

6. No caso da Tate, o acesso gratuito é assegurado na visita às exposições da colecção do museu, sendo que a entrada nas exposições temporárias é paga.

7. https://www.tate.org.uk/about-us/governance (consultado em Janeiro 6, 2021). 
8. Foi apenas em 2016 que o governo do Reino Unido publicou um novo white paper sobre cultura, no qual reforça a ideia de que todas as pessoas devem ter acesso à cultura, mas que as organizações financiadas com fundos públicos devem aumentar as oportunidades para os cidadãos menos privilegiados (Fisher 2020).

9. No final de 2019 foi apresentada a nova estratégia a dez anos do Arts Council, Let's Create: Strategy 2020-2030. https://www.artscouncil.org.uk/letscreate (consultado em Agosto 25, 2021).

10. Designado originalmente por Arts Council of Great Britain, em 1996 este organismo foi dividido em três entidades distintas, Arts Council England, Arts Council Wales e Scottish Arts Council. https://www.artscouncil.org.uk/timeline/1940s (consultado em Dezembro 10, 2020).

11. Este financiamento é resultado de um competitivo processo de candidatura, sendo que o apoio do ACE representa apenas uma parte do financiamento das instituições.

12. As Regional Arts Associations foram criadas na década de 1950 como forma de ligação entre o Arts Council e as províncias (regions) do Reino Unido.

13. A Tate é um órgão público executivo não-departamental e uma instituição de caridade, patrocinada e regulada pelo DCMS, e governada por um board of trustees. Maria Balshaw é, desde 2017, a Directora da Tate, assistida directamente por um grupo executivo composto por oito diretores, incluindo Anna Cutler, Directora de Learning and Research, nomeada em 2009 e responsável pela estratégia educativa das quatro galerias da Tate e das suas respectivas equipas. https://www.tate.org.uk/about-us/governance (consultado em Novembro 26, 2020).

14. Antes os únicos recursos educativos disponíveis eram palestras e visitas guiadas, iniciadas em 1900 (Tate Gallery 2020).

15. Ver, por exemplo, Situating Gallery Education (Allen 2008); Teaching in the Art Museum: Interpretation as Experience (Burnham e Kai-Kee 2011); Os Museus de Arte e a Educação: Discursos e Práticas Contemporâneas (Fróis 2008); From Knowledge to Narrative: Educators and the Changing Museums (Roberts 1997).

16. Este foi um dos projectos apoiados na sequência dos resultados apresentados no relatório Moving Culture: An Enquiry into the Cultural Activities of Young People (Willis 1990).

17. Posteriormente, o programa passou a reportar directamente à Tate e a seguir as directrizes do DCMS (Sayers 2014b).

18. Esther Sayers foi coordenadora do programa Raw Canvas e realizou a sua tese de doutoramento sobre o mesmo (Sayers 2014b).

19. Estas instituições fazem parte da Plus Tate, uma rede que integra actualmente 35 organizações ligadas às artes visuais no Reino Unido, com o intuito de partilhar ideias, práticas e recursos, e colaborar no desenvolvimento de programas conjuntos.

20. A segunda publicação referida foi editada com base na tese de doutoramento "Like Oil and Water"? Partnerships Between Visual Art Institutions and Youth Organisations (Sim 2018), produzida durante o projecto e financiada pelo Arts and Humanities Research Council (AHRC).

21. Ver, por exemplo, Room to Rise: The Lasting Impact of Intensive Teen Programs in Art Museums (Linzer e Munley 2015).

22. https://www.tate.org.uk/tate-collective/producers (consultado em Agosto 16, 2021).

23. Ver, por exemplo, The Value of the Blueprint Festival (Cawley-Gelling et al. 2015), um testemunho dos jovens membros do Tate Collective sobre um projecto desenvolvido na Tate Liverpool no âmbito do programa Circuit. 


\section{RESUMOS}

A participação dos jovens em museus, enquanto público independente, com interesses e motivações próprios, é um fenómeno recente. Nas últimas décadas observa-se, em particular no Reino Unido e nos EUA, um crescente investimento em programas para jovens entre os 15 e os 25 anos, com destaque para iniciativas continuadas, sustentadas numa abordagem de cocriação com e para jovens. Este artigo centra-se no desenvolvimento do programa para jovens na Tate Britain, Tate Modern, Tate Liverpool e Tate St Ives, identificando os principais momentos de torsão entre 1989 e 2019. Tem por base a análise de relatórios, policy briefs e outras publicações relevantes. Esta análise considera as mudanças nas políticas culturais e consequentes linhas de financiamento, públicas e privadas, no Reino Unido, com destaque para a tensão epistémica entre acesso e excelência, e o papel do Arts Council, da Fundação Calouste Gulkbenkian (UK Branch) e da Fundação Paul Hamlyn. Se o que enquadrou as primeiras iniciativas para jovens na Tate foi um discurso de responsabilização social perante grupos menos representados nos seus públicos, o caminho traçado ao longo dos últimos 30 anos foi de autonomização. 0 programa para jovens assume hoje um perfil de destaque, não só dentro da instituição como a nível nacional e internacional. A sua sustentabilidade alicerça-se numa abordagem de investigação participativa, central ao programa educativo da Tate, que permite traçar fluxos de influência, através dos quais a voz do museu e dos seus interlocutores - jovens, educadores, instituições parceiras -, podem ter eco nas práticas e políticas culturais futuras.

The participation of young people in museums, as an independent audience, with their own interests and motivations, is a recent phenomenon. In the last decades, in particular in the UK and the USA, there has been a growing investment in programmes targeting this age group, between 15 and 25, with prominence for long-term and peer-led initiatives - with and for young people. This paper focuses on the development of the youth programme in Tate Britain, Tate Modern, Tate Liverpool and Tate St Ives, identifying key moments between 1989 and 2019, based on the analyses of reports, policy briefs and other relevant publications. This analysis considers the shifts in the cultural policies and consequent public and private funding opportunities in the UK, highlighting the epistemic tension between access and excellence, and the role of the Arts Council, the Calouste Gulbenkian Foundation (UK Branch) and the Paul Hamlyn Foundation. Although the first initiatives for youth at Tate were influenced by a social responsibility agenda, concerned with misrepresented groups in museums, the journey that was drafted over the last 30 years was one of autonomy. Today the youth programme has a central profile not only within the institution but also nationally and internationally. Its sustainability is grounded on a practice-led research approach, central to Tate's education programme, that allows for fluxes of influence to be drawn, through which the voice of the museum and its interlocutors - young people, educators, partner institutions - can have an echo in the cultural practices and policies of the future.

\section{ÍNDICE}

Keywords: cultural policies and museums, Tate, museum and education, museums and young people, audience engagement

Palavras-chave: políticas culturais e museus, Tate, museus e educação, museus e jovens, públicos e mediação 


\section{AUTOR}

\section{CAROLINA SILVA}

É investigadora auxiliar no Instituto de Ciências Sociais (ICS) da Universidade de Lisboa, com uma bolsa Marie Skłodowska-Curie. Actualmente está a desenvolver o projecto "Educational Provision and Professional Training for Youth in Contemporary Art Museums" em parceria com o Museu de Arte, Arquitetura e Tecnologia (MAAT). É doutorada em Educational Studies pelo Goldsmiths, Universidade de Londres, com uma tese sobre colectivos de jovens em museus de arte contemporânea. Entre 2017 e 2020 foi curadora do programa para comunidades na Whitechapel Gallery, em Londres. Tem trabalhado e publicado sobre educação em museus, educação artística e cultura visual, práticas artísticas e pedagogias participativas.

Instituto de Ciências Sociais, Av. Professor Aníbal de Bettencourt, 9, 1600-189, Lisboa, Portugal, carolina.silva@ics.ulisboa.pt 